\title{
ARTICLE
}

\section{Local viscoelasticity of living cells measured by rotational magnetic spectroscopy}

J.-F. Berret ${ }^{1}$

When submitted to a magnetic field, micron-size wires with superparamagnetic properties behave as embedded rheometers and represent interesting sensors for microrheology. Here we use rotational magnetic spectroscopy to measure the shear viscosity of the cytoplasm of living cells. We address the question of whether the cytoplasm is a viscoelastic liquid or an elastic gel. The main result of the study is the observation of a rotational instability between a synchronous and an asynchronous regime of rotation, found for murine fibroblasts and human cancer cells. For wires of susceptibility 3.6, the transition occurs in the range $0.01-1 \mathrm{rads}^{-1}$. The determination of the shear viscosity (10-100 Pas) and elastic modulus ( $5-20 \mathrm{~Pa}$ ) confirms the viscoelastic character of the cytoplasm. In contrast to earlier studies, it is concluded that the interior of living cells can be described as a viscoelastic liquid, and not as an elastic gel.

\footnotetext{
${ }^{1}$ Matière et Systèmes Complexes, UMR 7057 CNRS Université Denis Diderot Paris-VII, Bâtiment Condorcet, 10 rue Alice Domon et Léonie Duquet, 75205 Paris, France. Correspondence and requests for materials should be addressed to J.-F.B. (email: jean-francois.berret@univ-paris-diderot.fr).
} 
$\mathrm{n}$ rheology, viscoelastic liquids and viscoelastic solids differ from each other by their stress relaxation function $G(t)$. In viscoelastic liquids, $G(t)$ is a decreasing function of the time which tends to zero as $t \rightarrow \infty$, whereas in viscoelastic solids $G(t)$ tends to a finite elastic modulus, indicating the presence of residual unrelaxed stresses even at very long time durations ${ }^{1}$. Hence, the static shear viscosity, which is defined as $\eta_{0}=\int_{0}^{\infty} G(t) d t$ takes a finite value for liquids and it is generally expressed as a product of a relaxation time and of an elastic modulus. For viscoelastic solids, the static viscosity is not defined, and the rheological properties are described in terms of a low frequency elastic modulus $G^{\prime}(\omega \rightarrow 0)$ and of a yield stress, that is, the critical value of the stress above which the sample does flow. In the following, viscoelastic solids will be termed 'elastic gels' for convenience. Here we address the question of the rheological properties of the intracellular medium of mammalian cells, and in particular the question to know whether the cytoplasm of such cells is a viscoelastic liquid or an elastic gel.

Rheometers are used to determine the relationship between the strain and stress on material sample of volume of the order of millilitres. Microrheology in contrast uses micron-size probes embedded in the material and needs much less sample, of the order of 1 picolitre. The past 20 years have seen increasingly rapid advances in this field, specifically in cell and tissue biomechanics $^{2,3}$. For living cells, techniques including atomic force microscopy $(\mathrm{AFM})^{4,5}$, optical and magnetic tweezers ${ }^{6-10}$, parallel microplates ${ }^{11}$ and active tracking of internalized particles ${ }^{7,10,12-14}$ were developed and provide consistent evaluation of the time or frequency dependencies of rheological parameters, such as the elastic compliance $J(t)$ or of the complex modulus $G^{\star}(\omega)$. Numerous studies performed on a wide variety of cell lines have shown that $J(t)^{9,14,15}$ and $G^{*}(\omega)^{8,9,12,14,16}$ obey scaling laws as a function of time or frequency, with exponents comprised between 0.1 and 0.5 . As for $G^{*}(\omega)$ measurements, the elastic modulus $G^{\prime}(\omega)$ was found to be larger than the loss modulus $G^{\prime \prime}(\omega)$ on broad frequency ranges. These results were interpreted as an indication that the mechanical response of the cell or of the cytoplasm is that of a weak elastic solid ${ }^{8-10,12,15,17}$, or in the terminology adopted previously that of an elastic gel. Out-of-equilibrium modelling suggests that the cell interior is a kind of soft glassy material with an effective temperature higher than that of the physiological temperature ${ }^{17}$. Alternative approaches of biomechanics were also attempted and provided different view points. On the basis of AFM micro-indentation and force-relaxation tests, Moeendarbary et $a l^{5}$ proposed that the cytoplasm behaves as a poroelastic material, where the water redistribution within the cytoplasm plays a fundamental role in setting the internal rheology. Other studies, in particular the earlier work of the Sackmann group ${ }^{6,7,18}$ suggest that the intracellular medium behaves like a liquid and is characterized by a finite value of the shear viscosity ${ }^{13}$. More recently, Kuimova et al. ${ }^{19,20}$ developed porphyrin-dimer-based molecular rotors in which fluorescence emitting frequencies scale with the viscosity of the surrounding medium. Evaluated on immortalized cervical cancer cells (HeLa cells), these authors found intracellular viscosities in the range $0.05-0.2 \mathrm{Pas}$. Chevry et al. show that micron-size wires internalized in mouse fibroblasts and MDCK epithelial cells exhibit Brownian-like orientational fluctuations, associated to finite effective viscosity of $0.1-1 \mathrm{Pas}$, in relative agreement with the determinations of Kuimova et al. ${ }^{21}$.

Regarding the complexity of the dynamics in living systems, and the contrasting results obtained so far, there is a need to develop new types of probes and protocols for testing the cell biomechanics. Following the pioneer work by Crick and
Hughes some 60 years ago $^{22}$, recent studies have shown that microrheology based on the tracking and monitoring of anisotropic probes could bring significant advances to the field ${ }^{23-34}$. It has been proposed, for example, that the shear viscosity of a fluid could be determined from the motion of a micro-actuator submitted to a rotating electric ${ }^{28}$ or magnetic field $^{27,32-36}$. These techniques are described as electric or magnetic rotational spectrometry ${ }^{29,30}$. With micro-actuators rotating at increasing frequency, a transition between a steady and a hindered motion is predicted for viscous fluids, and it is found experimentally $25,27,29,30,37,38$. A recent quantitative analysis shows that for viscoelastic liquids, the elasticity does not affect the onset of the instability and that the expression for the critical frequency remains the same as in a viscous fluid ${ }^{25}$. For an elastic gel of infinite static viscosity, in contrast, extrapolation yields a critical frequency going to zero, suggesting that the technique of the rotating wire is well adapted to differentiate liquid- from gel-like behaviours, as defined in rheology.

In the present paper, we exploit this concept to study the mechanical response of the cytoplasm of mouse fibroblast $\mathrm{NIH} / 3 \mathrm{~T} 3$ and human cancer HeLa cells. It is first demonstrated that the micron-size wires specifically fabricated for the study are nontoxic, enter spontaneously inside living cells and that they are not comprised in membrane-bound compartments. Actuated by a low-frequency external field, the wires rotate at the same angular speed than the field, whereas at higher frequency their motion exhibits back-and-forth oscillations. Values for the static viscosity, elastic modulus and relaxation time of the cytoplasm are derived from a complete analysis of the wire temporal trajectories. It is concluded that the interior of fibroblasts and HeLa cells can be appropriately described as viscoelastic liquids.

\section{Results}

Magnetic wire synthesis and characterization. Crystalline iron oxide nanoparticles were synthesized by co-precipitation of iron(II) and iron(III) salts in alkaline aqueous media and by further oxidation of the magnetite $\left(\mathrm{Fe}_{3} \mathrm{O}_{4}\right)$ into maghemite $\left(\gamma-\mathrm{Fe}_{2} \mathrm{O}_{3}\right)$. The size and dispersity (ratio between standard deviation and average diameter) of the particles prepared were determined from transmission electron microscopy $\left(D_{\mathrm{TEM}}=13.2 \mathrm{~nm}, s=0.23\right.$, Fig. 1 and Supplementary Fig. 1). Their crystallinity and structure was assessed by electron beam microdiffraction (Supplementary Fig. 2). The magnetization curves at different volume fractions were obtained by vibrating sample magnetometry and adjusted using a paramagnetic model ${ }^{39,40}$. The adjustment provided a specific magnetization of $3.5 \times 10^{5} \mathrm{~A} \mathrm{~m}^{-1}$ and a magnetic diameter of $10.7 \mathrm{~nm}$ (Supplementary Fig. 3). Light scattering was used to measure the weight-average molecular weight $\left(M_{w}=12 \times 10^{6} \mathrm{~g} \mathrm{~mol}^{-1}\right)$ and the hydrodynamic diameter $\left(D_{\mathrm{H}}=27 \mathrm{~nm}\right)$ for the bare particles ${ }^{40}$. Values of the electrophoretic mobility and zeta-potential were also derived $\left(\mu_{\mathrm{E}}=-3.8 \times 10^{-4} \mathrm{~cm}^{2} \mathrm{~V}^{-1}\right.$, $\zeta=-48 \mathrm{mV}$, Supplementary Table 1) and indicated that the dispersions were stabilized by electrostatic forces ${ }^{41}$.

For the wire synthesis, the particles were coated with poly(sodium acrylate) ${ }^{39,41}$ (Aldrich) of molecular weight $M_{w}=5,100 \mathrm{~g} \mathrm{~mol}^{-1}$. The wires were made according to a bottom-up co-assembly process using $\gamma$ - $\mathrm{Fe}_{2} \mathrm{O}_{3}$-coated particles and oppositely charged polyelectrolytes. The polymer used was poly(trimethylammoniumethylacrylate)-b-poly(acrylamide) with molecular weights $11,000 \mathrm{~g} \mathrm{~mol}^{-1}$ for the cationic block and $30,000 \mathrm{~g} \mathrm{~mol}^{-1}$ for the neutral block ${ }^{42,43}$. Supplementary Fig. 4 illustrates the protocol for the fabrication of the wires. Figure $1 \mathrm{~b}$ displays a TEM image of a single wire illustrating its nanostructure, whereas Fig. 1c shows a collection of wires 


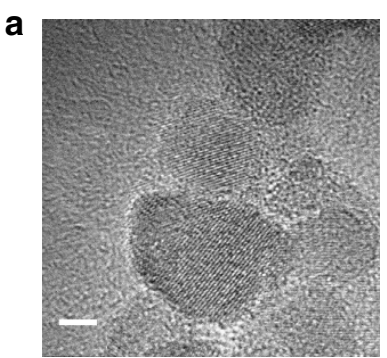

b

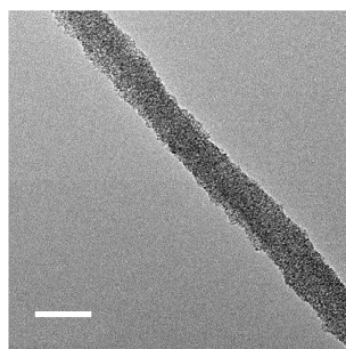

c

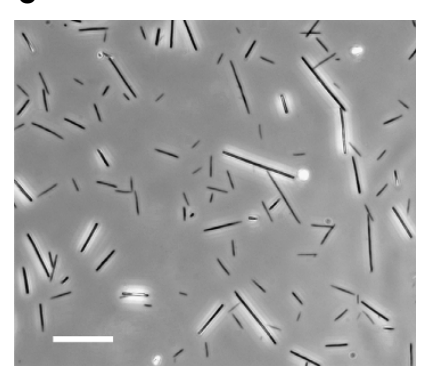

d

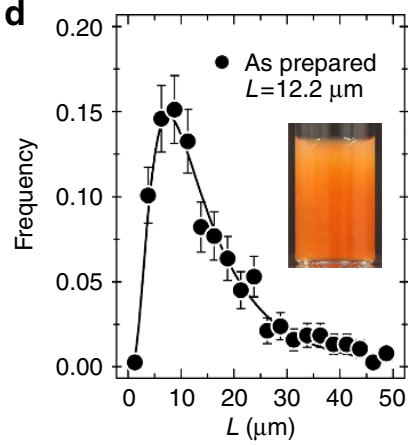

Figure 1 | Characterization of particles and wires. (a) High-resolution transmission electron microscopy (TEM) of $13.2 \mathrm{~nm}$ iron oxide nanoparticles (scale bar, $2 \mathrm{~nm}$ ). (b) TEM of magnetic wires made by co-assembly using oppositely charged particles and polyelectrolytes (scale bar, $200 \mathrm{~nm}$ ). (c) Phase-contrast image of magnetic wires deposited on a glass substrate and observed by optical microscopy ( $\times 60$, scale bar, $10 \mu \mathrm{m}$ ). (d) Size distribution of the wires adjusted using a log-normal distribution function with length of $12.2 \mu \mathrm{m}$ and dispersity of 0.70 . Error bars are defined as s.e.m. Inset: image of a vial containing a dispersion of wires at the concentration of $10^{6}$ wires $\left.\mu\right|^{-1}$.

deposited on a glass substrate and observed by optical microscopy $(40 \times)$. The as-prepared samples contained anisotropic objects of median length $12.2 \mu \mathrm{m}$ and of diameter $0.49 \mu \mathrm{m}$ (Fig. 1d). Before incubation with cells, the wires were sonicated for $120 \mathrm{~s}$ to shorten their lengths and sorted by magnetic separation. The sonicated wires had an average length of $2.4 \mu \mathrm{m}$ and a dispersity of 0.35 . The wires dispersion was then autoclaved (Tuttnauer Steam Sterilizer $2340 \mathrm{M}$ ) at $120^{\circ} \mathrm{C}$ and atmospheric pressure for 20 minutes to prevent bacterial contamination for cell culture, concentrated by magnetic sedimentation at $10^{6}$ wires $\mu \mathrm{l}^{-1}$ and stored at $4{ }^{\circ} \mathrm{C}$ in a secure environment before use.

Wire microrheology on model fluids and gels. First we derive the equation of motion of a wire immersed in well-known rheological models ${ }^{1}$ (Supplementary Fig. 5) and submitted to a rotating magnetic field, and then assess the validity of the technique by comparing the predictions to observations obtained on a wormlike surfactant solution and on a polysaccharide gel.

For a viscous liquid of viscosity $\eta_{0}$, a wire submitted to a rotating field experiences restoring a viscous torque that slows down its rotation. As a result, its motion undergoes a transition between a synchronous and an asynchronous rotation. The critical frequency $\omega_{\mathrm{C}}$ between these two regimes reads:

$$
\omega_{\mathrm{C}}=\frac{3}{8} \frac{\mu_{0} \Delta \chi}{\eta_{0}} g\left(\frac{L}{D}\right) \frac{D^{2}}{L^{2}} H^{2}
$$

where $\mu_{0}$ is the permeability in vacuum, $L$ and $D$ the length and diameter of the wire, $H$ the amplitude of the magnetic excitation and $g\left(\frac{L}{D}\right)=\operatorname{Ln}\left(\frac{L}{D}\right)-0.662+0.917 \frac{D}{L}-0.050\left(\frac{D}{L}\right)^{2}$ is a dimensionless function of the anisotropy ratio ${ }^{44,45}$. In equation (1), $\Delta \chi=\chi^{2} /(2+\chi)$ where $\chi$ denotes the magnetic susceptibility ${ }^{27}$. For data treatment, the geometrical characteristics are combined into the dimensionless parameter $L^{*}=L / D \sqrt{g(L / D)}$. Under these conditions, equation (1) becomes:

$$
\omega_{\mathrm{C}}=\frac{3 \mu_{0} \Delta \chi}{8 \eta_{0}} \frac{H^{2}}{L^{*^{2}}}
$$

The average angular velocity $\Omega(\omega)$ in the two regimes expresses as $^{27,35}$ :

$$
\begin{array}{cc}
\omega \leq \omega_{\mathrm{C}} & \Omega(\omega)=\omega \\
\omega \geq \omega_{\mathrm{C}} & \Omega(\omega)=\omega-\sqrt{\omega^{2}-\omega_{\mathrm{C}}^{2}}
\end{array}
$$

With increasing frequency, the average velocity increases linearly, passes through a cusp-like maximum at the critical frequency and then decreases. The transition between the synchronous and asynchronous regimes was used to calibrate the wire rheometer and determine the susceptibility parameter $\Delta \chi$. Experiments were performed at $T=26^{\circ} \mathrm{C}$ on a $85 \mathrm{wt}$. \% water-glycerol mixture of static viscosity $\eta_{0}=0.062 \mathrm{~Pa} \mathrm{~s}^{-1}$. In a purely viscous fluid, the critical frequency is found to decrease as $L^{*-2}$ in agreement with equation (2). For wires made from $13.2 \mathrm{~nm}$ particles and block copolymers, we found $\Delta \chi=2.3 \pm 0.7$, and a magnetic susceptibility $\chi=3.6 \pm 0.9$ (Supplementary Fig. 6). Here and in the following, the uncertainty is defined as s.e.m.

A Maxwell fluid is described by a spring and dashpot in series ${ }^{1}$. An actuated wire immersed in such a medium experiences a viscous and an elastic torque that both oppose the applied magnetic torque. The differential equation describing the wire motion has been derived and solved, leading to the following predictions (Supplementary Note 1 ). With increasing $\omega$, the wire undergoes the same type of transition as the one described previously and the critical frequency $\omega_{\mathrm{C}}$ expresses as in equation (1). The static viscosity $\eta_{0}$ in equation (1) is however replaced by the product $G \tau$, where $G$ and $\tau$ denote the shear elastic modulus and the relaxation time of the fluid. The set of equations in equation (3) is also identical to that of a Newtonian fluid. From the amplitude of the oscillations $\theta_{\mathrm{B}}(\omega)$ (more precisely the angle by which the wire returns back after a period of increase) in the asynchronous regime, it is possible to determine the shear elastic modulus $G$ using ${ }^{25}$ :

$$
\lim _{\omega \rightarrow \infty} \theta_{\mathrm{B}}(\omega)=\frac{3}{4} \frac{\mu_{0} \Delta \chi}{G} g\left(\frac{L}{D}\right) \frac{D^{2}}{L^{2}} H^{2}
$$

The above predictions were tested by monitoring the wire motion in a wormlike micellar solution made from cetylpyridinium chloride and sodium salicylate $([\mathrm{NaCl}]=0.5 \mathrm{M})$ at $2 \mathrm{wt}$. \% (ref. 46). The rheological parameters of the surfactant solution as determined by cone-and-plate rheometry were $\eta_{0}=1.0 \pm 0.1 \mathrm{Pas}, \quad G=7.1 \pm 0.1 \mathrm{~Pa} \quad$ and $\quad \tau=0.14 \pm 0.01 \mathrm{~s}$ (Supplementary Note 2). From the evolution of the average angular velocity $\Omega(\omega)$ of actuated wires and from the position of the maximum (equation (3)), a static viscosity of $1.3 \pm 0.3 \mathrm{~Pa} s$ was obtained. From the oscillation amplitudes in the regime $\omega \tau \gg 1$ (equation (4)), a modulus of $9.4 \pm 2 \mathrm{~Pa}$ was derived (Supplementary Fig. 7). Experiments performed with wires of different lengths and in various magnetic field conditions confirmed the good agreement with cone-and-plate rheometry, and demonstrates the ability of the technique to measure the linear viscoelasticity of a Maxwell fluid ${ }^{25}$.

A Kelvin-Voigt element aims to describe a viscoelastic solid, and it is represented by a spring and dashpot in parallel ${ }^{1}$. The resolution of the differential equation of motion of a wire leads to the following predictions (Supplementary Note 1). At all frequencies, the wire rotation exhibits back-and-forth oscillations 
at a frequency twice that of the field, and the average angular velocity $\Omega(\omega)=0$. In addition, the amplitude of the oscillations $\theta_{B}(\omega)$ varies inversely with the shear elastic modulus according to:

$$
\theta_{\mathrm{B}}(\omega)=\frac{3}{4} \frac{\mu_{0} \Delta \chi}{G^{\prime}(\omega)} g\left(\frac{L}{D}\right) \frac{D^{2}}{L^{2}} H^{2}
$$

Gellan gum (phytagel, Sigma-Aldrich), a linear anionic polysaccharide comprising glucose, glucuronic acid and rhamnose building units ${ }^{47}$ was added slowly to a $1 \mathrm{mM}$ calcium chloride solution at room temperature with rapid stirring before heating up to $50^{\circ} \mathrm{C}$. Samples prepared at concentrations $0.3,0.5,0.75,1$ and 2 wt. $\%$ and studied by cone-and-plate rheometry exhibited a gel-like behaviour: the elastic modulus exhibits scaling of the form $G^{\prime}(\omega) \sim \omega^{0.2}$ and $G^{\prime}(\omega)>G^{\prime \prime}(\omega)$ (Supplementary Note 3 ). At frequencies $5 \times 10^{-3}$ to $10 \mathrm{rad} \mathrm{s}^{-1}$, the rotation angle of the wires reveal steady oscillations, and an average angular speed $\Omega(\omega)$ equal to zero, in agreement with the Kelvin-Voigt predictions (Supplementary Fig. 8). Using equation (5), we found an elastic modulus $G^{\prime}(\omega)$ of $2.5 \pm 0.8 \mathrm{~Pa}$, $9.5 \pm 3 \mathrm{~Pa}$ and $95 \pm 30 \mathrm{~Pa}$ for the $0.3,0.5$ and 1 wt. \% samples, respectively, again in good agreement with the cone-and-plate data $\left(G^{\prime}(\omega)=3 \pm 0.5 \mathrm{~Pa}, \quad 13 \pm 2 \mathrm{~Pa}\right.$ and $\left.130 \pm 10 \mathrm{~Pa}\right)$. In conclusion of this part, we have found that theory correctly predicts the motion of rotating magnetic wires immersed in viscoelastic model systems and that the viscoelastic parameters retrieved, such as the viscosity or the elastic modulus are those of the linear shear rheology.

Localization of the wires inside living cells. Here we establish the experimental conditions under which the wires interact with the cells, and in which part of the cytoplasm they are located. The localization of the wires inside the cells was determined by transmission electron microscopy. The incubation time was $24 \mathrm{~h}$ and the amount of wires per cell was 10 for the two cell lines. Under such conditions, no perturbations of the cell morphology or cell cycle were observed (Supplementary Fig. 9). In a previous report, it was shown that the capture rate for neutral wires was $\sim 10 \%$ at $24 \mathrm{~h}$, leading to an average number of one wire per cell in the present experiment ${ }^{48}$. Figure 2a,b display TEM images of NIH/3T3 and HeLa cell interiors, respectively. The elongated objects appearing in the TEM images are in the range

$\mathrm{NIH} / 3 \mathrm{~T} 3$

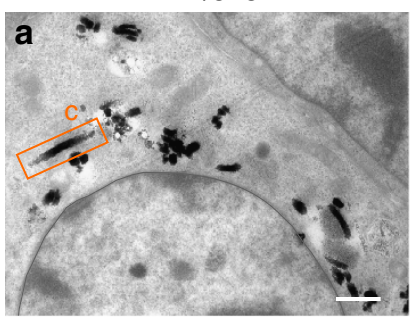

C

Figure 2 | Localization of wires inside living cells. Representative transmission electron microscopy image of (a) NIH/3T3 fibroblasts and (b) HeLa cancer cells incubated with $2.4 \mu \mathrm{m}$ wires for $24 \mathrm{~h}$ at the concentration of 10 wires per cell. For the fibroblasts, clusters in the range of $200 \mathrm{~nm}$ are also visible. Scale bars in $\mathbf{a}, \mathbf{b}$ are 1 and $2 \mu \mathrm{m}$, respectively. (c,d) Close-up views of regions marked by an orange rectangle in $\mathbf{a}, \mathbf{b}$ respectively, indicating that the wires are not enclosed in membrane-bound compartments. Scale bars are $200 \mathrm{~nm}$.
$200 \mathrm{~nm}-1 \mu \mathrm{m}$ and they are made of densely packed particles of high electronic contrast. These threads are pieces of wires that were taken up by the cells. Shorter than their initial lengths, the threads also have sharp and diffuse extremities. These observations are interpreted by assuming that elongated structures not in the plane of the microtome section were shortened during the sample preparation, an outcome that was already noted in previous studies ${ }^{48,49}$. In addition to wires, clusters in the range of $200 \mathrm{~nm}$ are also visible. These clusters result from the wire degradation occurring during incubation or the sample preparation. Close-up views of the TEM images in Fig. 2c,d indicate that the wires are not in membrane-bound compartments $^{50}$. This result was already pointed out in our previous study on fibroblasts. Here however, the TEM outcomes are similar for the cancerous HeLa cells and for human lymphoblasts, indicating that the absence of endosomal membrane around internalized wires may be specific to anisotropic objects. Concerning the entry mechanism, several scenarios were suggested, including the perforation of the cellular membrane $e^{51,52}$, macropinocytosis ${ }^{53}$ or multiple combined processes ${ }^{48,54}$. At this stage, no definite conclusion can be made concerning the entry mechanism into NIH/3T3 or HeLa cells. The absence of cytoplasmic membrane around the internalized probes ensures, however, that the wires will probe the mechanical properties of the cytosol.

Evidence of wire rotational instability in living cells. We then examined the motions of wires internalized into cells and submitted to a $14 \mathrm{mT}$ rotating field. In this part, emphasis is put on the time-dependent behaviour. Wires of lengths 2 to $6 \mu \mathrm{m}$ were studied as a function of the frequencies $\omega$. For each condition, a $100 \mathrm{~s}$ movie was recorded and digitalized, from which the position of the centre-of-mass and orientation angle of the wire were retrieved and plotted as a function of time. Figure $3 \mathrm{a}$ shows the rotation of a $2.8 \mu \mathrm{m}$ wire internalized into a fibroblast at the angular frequency of $0.14 \mathrm{rad} \mathrm{s}^{-1}$ and Fig. $3 b$ illustrates the time dependence of the angle $\theta(t)$ at different frequencies (see Supplementary Movie 1). For the conditions tested, $\theta(t)$ increases linearly with time, and the slope $\Omega=\langle d \theta(t) / d t\rangle_{\mathrm{t}}$ corresponds exactly to the actuating frequency. Here, $\langle\ldots\rangle_{t}$ denotes the time average in the steady regime. In this first regime, the wire rotates with the field, and $\theta(t)=\omega t$.

Figure $3 c$,d illustrate the change of regime as the angular frequency is increased above a critical value, here $\omega_{\mathrm{C}}=0.15$ $\mathrm{rad} \mathrm{s}^{-1}$. The microscopy images on the left hand side show that after a clockwise steady rotation (between 23.8 and $26.4 \mathrm{~s}$ ), the wire comes back rapidly by 50 degrees in an anticlockwise motion, indicating that the wire rotation is hindered. On a longer period, the wires are animated with back-and-forth motion characteristic of the asynchronous regime (Fig. 3d). The traces recorded at $\omega=0.41,0.76$ and $1.87 \mathrm{rads}^{-1}$ display steady oscillations, with a rotation angle that continues to increase with time (see Supplementary Movies 2 and 3). In this second regime, however, it is observed that $\Omega(\omega) \ll \omega$. The data shown in the figure are representative of the overall behaviour found for wires in living fibroblast and HeLa cells. In these time-resolved experiments, it was verified that the centre-of-mass of the wires remained punctual during the steady or hindered rotations, insuring that the same volume of the cytosol is probed during the measurements. In conclusion to this part, with increasing frequency, wires dispersed in the intracellular medium of fibroblasts or of Hela cells undergo a transition between a synchronous and asynchronous regime. The critical frequency is found in the range $0.01-1 \mathrm{rads}^{-1}$. 

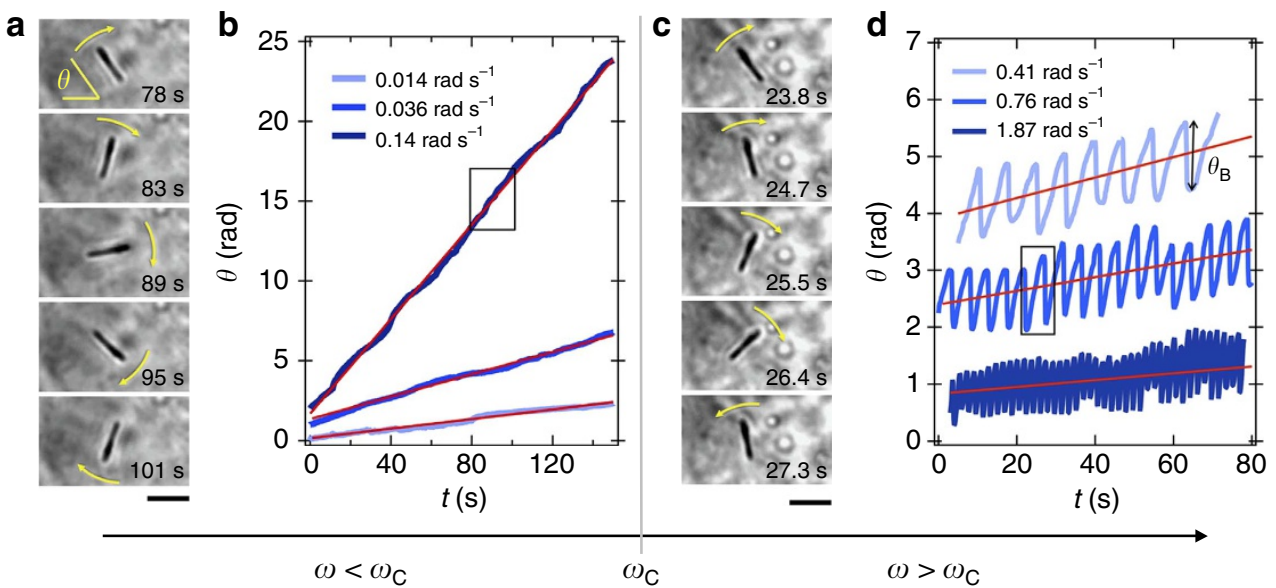

Figure 3 | Evidence of wire rotational instability in living cells. (a) Optical microscopy images of a $2.8 \mu \mathrm{m}$ internalized wire subjected to a rotating field of $14 \mathrm{mT}$ and the frequency of $0.14 \mathrm{rad} \mathrm{s}^{-1}$. (b) Time dependences of the angle $\theta(t)$ at varying frequencies $\omega=0.014,0.036$ and $0.14 \mathrm{rad} \mathrm{s}^{-1}$. The rectangle around $80 \mathrm{~s}$ provides the time range for the images on the left hand side. The straight lines in red were calculated from the expression $\theta(t)=\omega t$, indicating that the wire rotates synchronously with the field, and at the same angular frequency. The synchronous rotation of the wire is illustrated in Supplementary Movie 1. (c,d) Same representation as in $\mathbf{a}, \mathbf{b}$ but for angular frequencies $\left(0.41,0.76\right.$ and $1.87 \mathrm{rads}^{-1}$ ) above the critical frequency $\omega_{\mathrm{C}}=0.15 \mathrm{rads} \mathrm{s}^{-1}$. The microscopy images on the left-hand side shows that after a clockwise rotation, the wire comes back rapidly in an anti-clockwise motion, indicating that the wire rotation is hindered (see Supplementary Movies 2 and 3. Scale bars in $\mathbf{a}, \mathbf{c}$ are $2 \mu \mathrm{m}$.
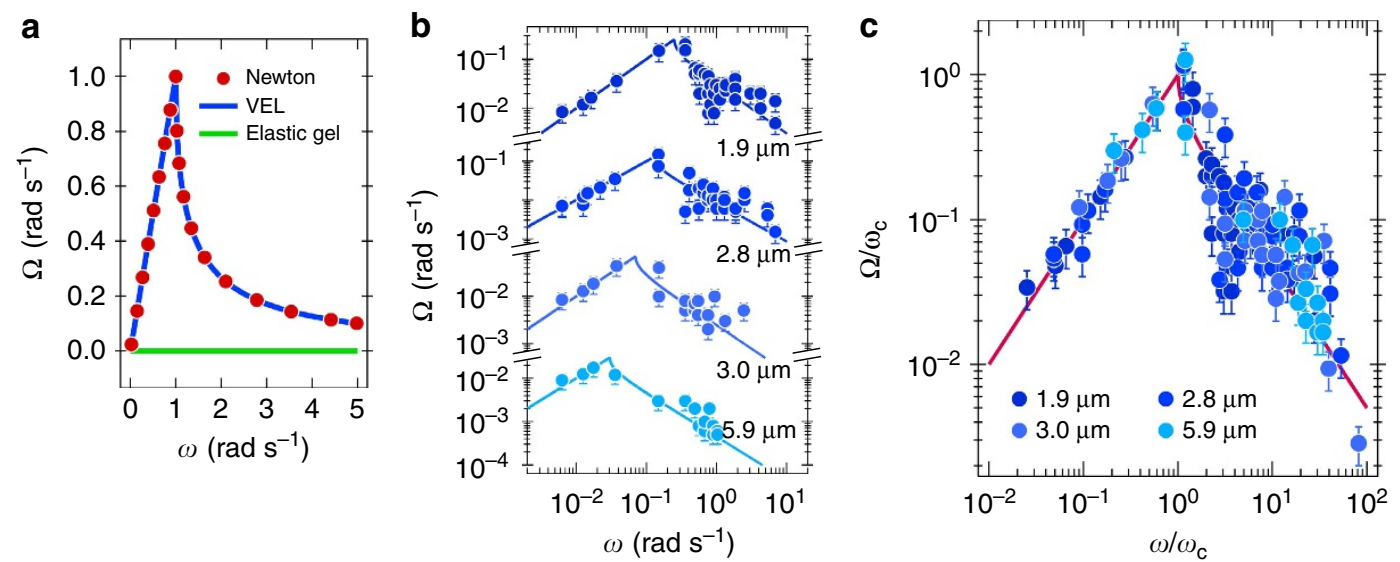

Figure 4 | Master curve for wire average rotation velocity in cells. (a) Average rotation velocity $\Omega(\omega)$ of a magnetic wire calculated for a Newtonian fluid, for a viscoelastic liquid (indicated as VEL) and for an elastic gel according to a set of models listed in Supplementary Note 1. For the sake of convenience, the viscous and the viscoelastic liquids have here the same static viscosity and $\omega_{\mathrm{C}}=1 \mathrm{rads}^{-1}$. (b) $\Omega(\omega)$-evolution for wires of lengths between 1.9 and $5.9 \mu \mathrm{m}$ internalized in the cytoplasm of mouse fibroblasts. The continuous lines (from equation (1)) indicate the existence of a critical frequency in the rotation dynamics. (c) Data from Fig. $4 \mathrm{~b}$ plotted in reduced units, $\Omega / \omega_{\mathrm{C}}$ versus $\omega / \omega_{\mathrm{C}}$. The continuous lines are $\Omega(X)=X$ and $\Omega(X)=X-\sqrt{X^{2}-1^{2}}$ for $X \leq 1$ and $X \geq 1$, respectively. Error bars in $\mathbf{b}, \mathbf{c}$ are defined as s.e.m.

Master curve for the wire average rotation velocity. To elucidate the rheological profile of NIH/3T3 and HeLa intracellular medium, the $\theta(t)$-traces of internalized wires were analysed and translated into a set of two parameters: the average rotation velocity $\Omega(\omega)$ and the amplitude $\theta_{\mathrm{B}}(\omega)=\left\langle\theta_{\mathrm{B}}(t, \omega)\right\rangle_{\mathrm{t}}$ of the oscillations in the unstable regime. As mentioned in the section on the validity of the magnetic wire spectroscopy, it was found that for Newton, Maxwell and Kelvin-Voigt models, $\Omega(\omega)$ and $\theta_{\mathrm{B}}(\omega)$ display specific asymptotic behaviours as a function of the frequency ${ }^{25}$. For the viscous and viscoelastic liquids, the average frequency $\Omega(\omega)$ is superimposed and show a cusp-like maximum at $\omega_{\mathrm{C}}$, whereas for an elastic gel the average rotation velocity is constant and equal to 0 . These different behaviours are illustrated in Fig. 4a.

Figure $4 \mathrm{~b}$ displays the $\Omega(\omega)$-evolution for wires of lengths between 1.9 and $5.9 \mu \mathrm{m}$. With increasing frequency, the average velocity increases linearly, passes through a maximum at $\omega_{\mathrm{C}}$ and then decreases. As already mentioned, the transition corresponds to the change of rotation regime, from synchronous rotation to back-and-forth oscillations. Note that the cut-off frequency increases as the length of the wire decreases (Fig. $4 \mathrm{~b}$ ). The data in Fig. 4 were adjusted using equation $(3)^{27,35}$. In both regimes, the agreement between the data and the model is excellent. At high frequency, the $\Omega(\omega)$-data exhibit some scattering that could come from time-dependent effects of the viscosity, and be related to the cellular activity. Table 1 provides the values of the critical frequency and static viscosity for the experiments in Fig. 4b. There, $\omega_{\mathrm{C}}$ varies from 0.03 to $0.2 \mathrm{rads}^{-1}$, and is associated to static viscosities between 20 and $80 \mathrm{~Pa}$ s. Figure $4 \mathrm{c}$ displays the data of Fig. $4 \mathrm{~b}$ in reduced units, $\Omega / \omega_{C}$ versus $\omega / \omega_{C}$. In this representation, the $\Omega / \omega_{\mathrm{C}}$-data are found to collapse onto the same master curve, now observed over four decades in $\omega$. The 
Table 1 | Viscoelastic parameters of NIH/3T3 fibroblast.

\begin{tabular}{|c|c|c|c|c|}
\hline Wire length & $\omega_{\mathrm{c}}\left(\operatorname{rad~s}{ }^{-1}\right)$ & $\eta_{\mathrm{o}}(\mathrm{Pa} \mathrm{s})$ & $\lim _{\omega \rightarrow \infty} \theta_{\mathbf{B}}(\omega)(\mathrm{rad})$ & $G(P a)$ \\
\hline$L=1.9 \mu \mathrm{m}$ & $0.20 \pm 0.05$ & $32 \pm 8$ & $0.8 \pm 0.1$ & $16 \pm 2$ \\
\hline$L=2.8 \mu \mathrm{m}$ & $0.15 \pm 0.02$ & $26 \pm 5$ & $0.8 \pm 0.1$ & $9 \pm 2$ \\
\hline$L=3.0 \mu \mathrm{m}$ & $0.07 \pm 0.02$ & $59 \pm 15$ & $0.8 \pm 0.1$ & $11 \pm 2$ \\
\hline
\end{tabular}

$\omega_{\mathrm{C}}$ denotes the critical cut-off frequency between the synchronous and asynchronous regimes for the data in Fig. $4 \mathrm{~b} . \eta_{\mathrm{O}}$ is the static viscosity derived from equation (1), lim $\theta_{\mathrm{B}}(\omega)$ is the angle by which the wire returns back at high frequency and $G$ is the elastic modulus obtained from equation (4). The error bars are defined as s.e.m.
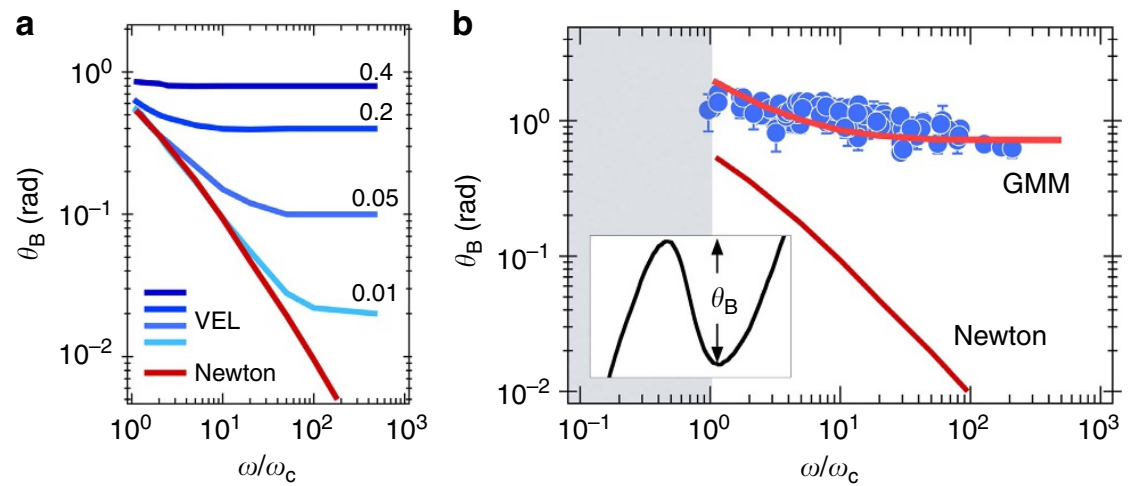

Figure 5 | Master curve for wire oscillation amplitude in cells. (a) Average rotation velocity $\Omega(\omega)$ of a magnetic wire calculated for a Newtonian fluid and for a viscoelastic liquid (indicated as VEL) of relaxation times ranging from $0.01 / \omega_{\mathrm{C}}$ (light blue) to $0.4 / \omega_{\mathrm{C}}$ (dark blue). (b) Variation of $\theta_{\mathrm{B}}$ as a function of the reduced frequency $\omega / \omega_{C}$ for wires of lengths 1.9 to $5.9 \mu \mathrm{m}$. The continuous lines were obtained from the Newtonian and from the Generalized Maxwell (GMM) models. Inset: definition of $\theta_{\mathrm{B}}$. Error bars in $\mathbf{b}$ are defined as s.e.m.

agreement obtained over a large frequency window attests to the reliability of the model. A comparison with the predictions shown in Fig. 4a suggests that the rheology of the intracellular medium is that of a viscous or of a viscoelastic liquid. The data in Fig. 4c also rule out the hypothesis of a gel-like rheology.

Master curve for the wire oscillation amplitude. Figure $5 \mathrm{a}$ displays the behaviours of the oscillation amplitudes for Newtonian and Maxwell fluids as a function of the reduced frequency $\omega / \omega_{\mathrm{C}} . \theta_{\mathrm{B}}$ being related to the asynchronous regime, it is defined only for $\omega / \omega_{C} \geq 1$. For the viscoelastic predictions (labelled VEL), the curves were calculated for different relaxation times ranging from $0.01 / \omega_{\mathrm{C}}$ to $0.4 / \omega_{\mathrm{C}}$, as indicated in the margin. For the Newtonian fluid, the amplitude of the oscillations decreases with increasing frequency. In contrast, the Maxwell fluid displays a crossover between a viscous and an elastic regime that occurs at a fixed value of the reduced frequency, here $\omega \tau=1 / 2$. The value of $1 / 2$ is explained by the fact that the frequency of the oscillations in this regime is twice that of the field. The existence of a plateau at high frequency is related to the elastic response of the fluid, and its height is given by equation (4) (ref. 21).

Figure $5 \mathrm{~b}$ shows the dependence of $\theta_{\mathrm{B}}$ in NIH/3T3 fibroblasts as a function of the reduced frequency for different wires of lengths between 1.9 and $5.9 \mu \mathrm{m}$. The major result that emerges from the figure is that the angles $\theta_{B}$ collected over many experimental conditions (that is, changing $\omega$ and $L$ ) are well superimposed when plotted against the reduced frequency. Starting at $\theta_{\mathrm{B}}=1.5 \mathrm{rad}$ slightly above $\omega_{\mathrm{C}}$, the angle exhibits a moderate but steady decrease with increasing $\omega$. Also displayed in the figure is the prediction for a Newtonian fluid, in which decrease is much stronger and cannot account for the data found in cells. Attempts to use a single-mode Maxwell model (Fig. 5a) were also inconclusive, and the $\theta_{\mathrm{B}}$-data could not be reproduced. The adjustment was noticeably improved using a Generalized Maxwell Model (noted GMM on the figure) and assuming a constant distribution of relaxation times. The spread of the distribution was found to go from $1 / \omega_{C}$ to $100 / \omega_{C}$. For $\omega_{C}$-values of $0.1 \mathrm{rads}^{-1}$, the time distribution extends hence from 0.1 to $10 \mathrm{~s}$. From this fitting, the elastic modulus $G$ of the cell interior was estimated. We found an elastic modulus $G=12 \mathrm{~Pa}$ (with a s.d. of $4 \mathrm{~Pa}, n=9$ ) for the fibroblasts and $G=14 \mathrm{~Pa}$ (with a s.d. of $9 \mathrm{~Pa}, n=10$ ) for the HeLa cells. Such values for the elasticity are in agreement with the results obtained by tracking techniques, specifically those probing the interior of the cell ${ }^{13}$. In contrast, $G$-values found here are one to two decades smaller than that determined by techniques probing the mechanical response of the entire cell, or that of the cell surface ${ }^{4,5,9}$. Such differences were already discussed in the literature, and for the latter experiments they were attributed to the cortical actin network ${ }^{12}$.

Shear viscosity of the cytoplasm. The model of rotating wires in a viscoelastic liquid contains an important prediction: the critical frequency $\omega_{\mathrm{C}}$ varies inversely with the square of the wire length. This relationship was verified for fluids of known viscosity 25,27 and tested again here for a $85 \%$ glycerol/water mixture (Supplementary Fig. 6). Figure 6a,b display the dependences of the critical frequency $\omega_{C}$ as a function of the reduced wire length $L^{*}=L / D \sqrt{g(L / D)}$ for the two cell lines studied. Least square calculations using a power law dependence of the form $\omega_{\mathrm{C}}\left(L^{*}\right) \sim L^{* \alpha}$ provide exponents $\alpha=-3.0 \pm 0.5$ and $\alpha=-6.5 \pm 1.0$ for $\mathrm{NIH} / 3 \mathrm{~T} 3$ and $\mathrm{HeLa}$, respectively (straight lines in red in Fig. 6). Two related effects could account for the discrepancy with respect to equation (2): the heterogeneities of the intracellular medium, which also contribute to a relatively broad scattering of the data points, and the possible dependence 

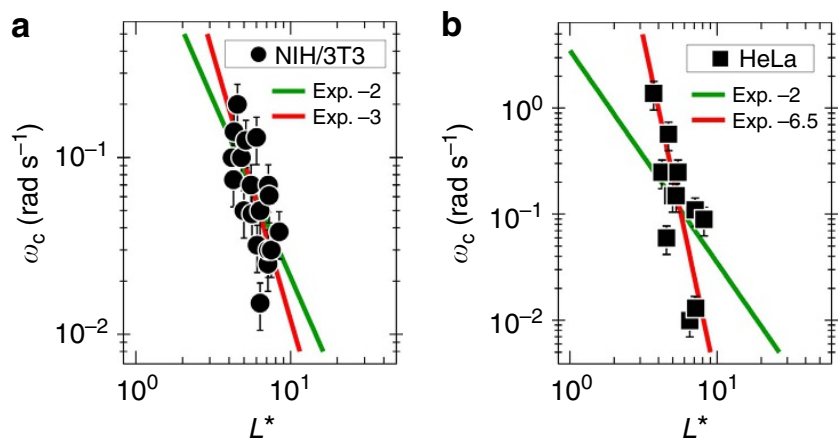

Figure 6 | Scaling critical frequency and wire length. Variation of the critical frequency $\omega_{C}$ as a function of the reduced wire length $L^{*}=L / D \sqrt{g(L / D)}$ for (a) NIH/3T3 mouse fibroblasts and (b) for HeLa cervical cancer cells. Straight lines in red are least-square fits using power laws with exponent (exp.) -3 and -6.5 , respectively, whereas straight lines in green are obtained from equation (2). Error bars are defined as s.e.m.

of the mechanical response on the length scale. This later feature was observed in active gels of F-actin using two-particle microrheology experiments ${ }^{55}$. By forcing the exponent to be -2 and repeating the fitting procedure, the static shear viscosity of fibroblasts could be estimated, providing $\eta_{0}=47(+26 /$ -14) Pas. This value is larger than those reported using passive microrheology in different culture and probe conditions $s^{13,21}$. For the Hela cells, the deviation between the observed and predicted scaling is significant, and $\eta_{0}$ could not be determined. This result confirms the high sampling variability of rheological parameters associated with this cell line.

\section{Discussion}

In this work, we used magnetic wires between 2 and $6 \mu \mathrm{m}$ to measure the static viscosity of living cells. The technique, known as rotational magnetic spectroscopy, is based on the experimental determination of a critical frequency $\omega_{\mathrm{C}}$ between two different rotation regimes, one where the wire and the field are synchronous, and one where the wire performs back-and-forth oscillations. The measure of the viscosity is obtained directly from the critical frequency $\omega_{C}$, through the relationship given by equation (1). This wire-based technique is known for more than 20 years but was applied only recently to complex fluids composed of colloids of synthetic and natural origin $n^{25,27,29,30,37,38,56}$. To the best of our knowledge, it was not applied yet to living cells. In a first step, we assess the validity of the wire-based microrheology technique using known rheological models. We have found that theory and experiments agree well with each other, and that the viscoelastic parameters retrieved (including the static viscosity $\eta_{0}$ and the elastic modulus $G$ ) are those of the linear shear rheology. With living cells, one of the key results here is that the rotational instability as in the range $10^{-2}-1 \mathrm{rads}^{-1}$ for the wires of magnetic susceptibility $\chi=3.6$. It is, therefore, accessible to standard rotating field devices mounted on an optical microscopy platform. In this paper, the analysis has been extended to the amplitude of the oscillations in the asynchronous regime and to its frequency dependence and scaling. The high frequency limit was used to estimate the shear elastic modulus $G$ of the intracellular medium for the NIH/3T3 fibroblasts and for the HeLa cervical cancer cells. The modulus is in the range 5-20 Pa. The determinations of the viscosity and elasticity confirm the viscoelastic character of the cytoplasm. However here, and in stark contrast to several studies, 90,56 , we conclude that the interior of living cells is best described in terms of a viscoelastic liquid, and not of an elastic gel. The conclusion was made possible by gaining access to a frequency range not explored before (down to $6 \times 10^{-3} \mathrm{rad} \mathrm{s}^{-1}$ ), and for which the cytoplasm was shown to flow. The present magnetic rotational spectroscopy method does not allow to determine the distribution of relaxation times with accuracy, in part because of the restricted range of frequencies explored. An estimate is nevertheless provided and it is found that the time distribution is constant in the range $0.1-10 \mathrm{~s}$ (ref. 9). The rheological model used to fit the data is in agreement with a Generalized Maxwell model combined with a distribution of relaxation times. In conclusion, the present work shows the potential of the wire-based magnetic rotation spectroscopy as an accurate rheological technique to distinguish between flow and yield stress behaviours in highly confined environment.

\section{Methods}

Microrheology set-up. Bright field microscopy was used to monitor the actuation of the wires as a function of time. Stacks of images were acquired on an IX73 inverted microscope (Olympus) equipped with a $\times 100$ objective. For the measure of the magnetic properties of the wires, $65 \mu \mathrm{l}$ of a dispersion of a know viscosity were deposited on a glass plate and sealed to a Gene Frame (Abgene/Advanced Biotech) dual adhesive system. The glass plate was introduced into a homemade device (Supplementary Fig. 10) generating a rotational magnetic field, thanks to two pairs of coils working with a $90^{\circ}$ phase shift. An electronic set-up allowed measurements in the frequency range $10^{-2}-100 \mathrm{rads}^{-1}$ and at magnetic fields $B=0-20 \mathrm{mT}$. A stream of air directed toward the measuring cell was used to thermalize the sample at $T=26$ or $37^{\circ} \mathrm{C}$. The image acquisition system consisted of an EXi Blue CCD camera (QImaging) working with Metaview (Universal Imaging Inc.). Images of wires were digitized and treated by the ImageJ software and plugins (http://rsbweb.nih.gov/ij/). The three-dimensional motion was extracted from their two-dimensional projection according a procedure described previously ${ }^{26,44}$. In brief, the out-of-plane motion of the wires is deduced from their projection on the focal plane of an optical microscope objective. An angular variable reflecting the out-of-plane motion is computed from the apparent wire length and its projected angular displacement.

Calibration of the wire-based rheometer. To determine the susceptibility parameter $\Delta \chi$ in equations (1) and (2), steady rotation experiments were carried out on a fluid of known viscosity. An $85 \mathrm{wt}$. \% glycerol-water mixture of static viscosity $\eta_{0}=0.062 \mathrm{~Pa} \mathrm{~s}^{-1}\left(\mathrm{~T}=26^{\circ} \mathrm{C}\right)$ was used as suspending medium. In a typical optical microscopy experiment, a wire was first selected, its length and diameter measured with the objective $\times 100$ and it was then submitted to rotation frequency sweep from 0.1 to $100 \mathrm{rad} \mathrm{s}^{-1}$. For a statistically relevant sample, the protocol was applied on 44 wires of lengths $2-20 \mu \mathrm{m}$ and at a magnetic field of $7 \mathrm{mT}$. In Supplementary Fig. 10, $\omega_{\mathrm{C}}$ is shown as a function of $L^{*}$. The critical frequency was found to decrease as $\omega_{\mathrm{C}} \sim L^{*-2}$, in agreement with the prediction of equation (2). From the prefactor $\left(3 \mu_{0} \Delta \chi H^{2} / 8 \eta_{0}=590 \pm 180 \mathrm{rad} \mathrm{s}^{-1}\right)$, we infer $\Delta \chi=2.3 \pm 0.7$, and $\chi=3.6 \pm 0.9$. Knowing $\Delta \chi$, a wire submitted to a rotating field will be subjected to a known magnetic torque, a condition that is a prerequisite for quantitative rotational microrheology.

Cell culture. NIH/3T3 fibroblast (ATCC-CRL-1658) and HeLa (ATCC-CCL-2) cells were grown in T25-flasks as a monolayer in DMEM with high glucose $\left(4.5 \mathrm{gl}^{-1}\right)$ and stable glutamine (PAA Laboratories $\mathrm{GmbH}$, Austria). The medium was supplemented with $10 \%$ fetal bovine serum and $1 \%$ penicillin/streptomycin (PAA Laboratories $\mathrm{GmbH}$ ), referred to as cell culture medium. Exponentially growing cultures were maintained in a humidified atmosphere of $5 \% \mathrm{CO}_{2}$ and $95 \%$ air at $37^{\circ} \mathrm{C}$, and in these conditions, the plating efficiency was $70-90 \%$ and the cell duplication time was $12-14 \mathrm{~h}$. Cell cultures were passaged twice weekly using trypsin-EDTA (PAA Laboratories $\mathrm{GmbH}$ ) to detach the cells from their culture flasks and wells. The cells were pelleted by centrifugation at $260 \mathrm{~g}$ for $5 \mathrm{~min}$. The supernatant was removed and cell pellets were re-suspended in assay medium and counted using a Malassez counting chamber.

Transmission electron microscopy. NIH/3T3 fibroblast cells were seeded onto the six-well plate. After a 24 -h incubation with $4 \mu \mathrm{m}$ wires, the excess medium was removed. The cells were washed in $0.2 \mathrm{M}$ phosphate buffer (phosphate-buffered saline, PBS) and fixed in $2 \%$ glutaraldehyde-phosphate buffer $(0.1 \mathrm{M})$ for $1 \mathrm{~h}$ at room temperature. Fixed cells were further washed in $0.2 \mathrm{M}$ PBS. The cells were then postfixed in a $1 \%$ osmium-phosphate buffer for $45 \mathrm{~min}$ at room temperature in dark conditions. After several washes with $0.2 \mathrm{M}$ PBS, the samples were dehydrated by addition of ethanol. Samples were infiltrated in 1:1 ethanol:epon resin for $1 \mathrm{~h}$ and finally in $100 \%$ epon resin for $48 \mathrm{~h}$ at $60^{\circ} \mathrm{C}$ for polymerization. Ninety-nanometre-thick sections were cut with an ultramicrotome (LEICA, Ultracut UCT) and picked up on copperrhodium grids. They were then stained for $7 \mathrm{~min}$ in $2 \%$-uranyl acetate and for $7 \mathrm{~min}$ in $0.2 \%$-lead citrate. Grids were analysed with a transmission electron microscope (ZEISS, EM 912 OMEGA) equipped with a $\mathrm{LaB}_{6}$ filament, at $80 \mathrm{kV}$. Images were recorded with a digital camera (SS-CCD, Proscan $1024 \times 1024)$, and the iTEM software. 


\section{References}

1. Larson, R. G. The Structure and Rheology of Complex Fluids (Oxford Univ. Press, 1998).

2. Bao, G \& Suresh, S. Cell and molecular mechanics of biological materials. Nat. Mater. 2, 715-725 (2003).

3. Squires, T. M. \& Mason, T. G. Fluid mechanics of microrheology. Annu. Rev. Fluid Mech. 42, 413-438 (2010).

4. Park, S., Koch, D., Cardenas, R., Kas, J \& Shih, C. K. Cell motility and local viscoelasticity of fibroblasts. Biophys. J. 89, 4330-4342 (2005).

5. Moeendarbary, E. et al. The cytoplasm of living cells behaves as a poroelastic material. Nat. Mater. 12, 253-261 (2013).

6. Bausch, A. R., Ziemann, F., Boulbitch, A. A., Jacobson, K. \& Sackmann, E. Local measurements of viscoelastic parameters of adherent cell surfaces by magnetic bead microrheometry. Biophys. J. 75, 2038-2049 (1998).

7. Bausch, A. R., Moller, W. \& Sackmann, E. Measurement of local viscoelasticity and forces in living cells by magnetic tweezers. Biophys. J. 76, 573-579 (1999).

8. Fabry, B. et al. Scaling the microrheology of living cells. Phys. Rev. Lett. 87, 148102 (2001)

9. Balland, M. et al. Power laws in microrheology experiments on living cells: comparative analysis and modeling. Phys. Rev. E 74, 17 (2006).

10. Guo, M. et al. Probing the stochastic, motor-driven properties of the cytoplasm using force spectrum microscopy. Cell 158, 822-832 (2014).

11. Mitrossilis, D. et al. Real-time single-cell response to stiffness. Proc. Natl Acad. Sci. USA 107, 16518-16523 (2010).

12. Hoffman, B. D., Massiera, G., Van Citters, K. M. \& Crocker, J. C. The consensus mechanics of cultured mammalian cells. Proc. Natl Acad. Sci. USA 103, 10259-10264 (2006).

13. Panorchan, P. et al. in Cell Mechanics (eds Wang, Y. L. \& Discher, D. E.) (Elsevier Academic Press Inc, 2007).

14. Weihs, D., Mason, T. G. \& Teitell, M. A. Bio-microrheology: a frontier in microrheology. Biophys. J. 91, 4296-4305 (2006).

15. Lenormand, G., Millet, E., Fabry, B., Butler, J. P. \& Fredberg, J. J. Linearity and time-scale invariance of the creep function in living cells. J. R. Soc. Interface 1, 91-97 (2004).

16. Deng, L. H. et al. Fast and slow dynamics of the cytoskeleton. Nat. Mater. 5, 636-640 (2006).

17. Gallet, F., Arcizet, D., Bohec, P. \& Richert, A. Power spectrum of out-ofequilibrium forces in living cells: amplitude and frequency dependence. Soft Matter 5, 2947-2953 (2009)

18. Feneberg, W., Westphal, M. \& Sackmann, E. Dictyostelium cells' cytoplasm as an active viscoplastic body. Eur. Biophys. J. Biophys. 30, 284-294 (2001).

19. Kuimova, M. K., Yahioglu, G., Levitt, J. A. \& Suhling, K. Molecular rotor measures viscosity of live cells via fluorescence lifetime imaging. J. Am. Chem. Soc. 130, 6672-6673 (2008)

20. Kuimova, M. K. et al. Imaging intracellular viscosity of a single cell during photoinduced cell death. Nat. Chem. 1, 69-73 (2009).

21. Chevry, L., Colin, R., Abou, B. \& Berret, J. F. Intracellular micro-rheology probed by micron-sized wires. Biomaterials 34, 6299-6305 (2013).

22. Crick, F. H. C. \& Hughes, A. F. W. The physical properties of cytoplasm-a study by means of the magnetic particle method .1. Experimental. Exp. Cell Res. 1, 37-80 (1950).

23. Celedon, A., Hale, C. M. \& Wirtz, D. Magnetic manipulation of nanorods in the nucleus of living cells. Biophys. J. 101, 1880-1886 (2011).

24. Chang, W. S., Ha, J. W., Slaughter, L. S. \& Link, S. Plasmonic nanorod absorbers as orientation sensors. Proc. Natl Acad. Sci. USA 107, 2781-2786 (2010).

25. Chevry, L., Sampathkumar, N. K., Cebers, A. \& Berret, J. F. Magnetic wire-based sensors for the microrheology of complex fluids. Phys. Rev. E Stat. Nonlin. Soft Matter Phys. 88, 062306 (2013).

26. Colin, R., Chevry, L., Berret, J.-F. \& Abou, B. Rotational microrheology of Maxwell fluids using micron-sized wires. Soft Matter 10, 1167-1173 (2014).

27. Frka-Petesic, B. et al. Dynamics of paramagnetic nanostructured rods under rotating field. J. Magn. Magn. Mater. 323, 1309-1313 (2011).

28. Pedaci, F., Huang, Z. X., van Oene, M., Barland, S. \& Dekker, N. H. Excitable particles in an optical torque wrench. Nat. Phys. 7, 259-264 (2011).

29. Tokarev, A. et al. Probing viscosity of nanoliter droplets of butterfly saliva by magnetic rotational spectroscopy. Appl. Phys. Lett. 102, 033701 (2013).

30. Tokarev, A., Luzinov, I., Owens, J. R. \& Kornev, K. G. Magnetic rotational spectroscopy with nanorods to probe time-dependent rheology of microdroplets. Langmuir 28, 10064-10071 (2012).

31. Zhang, L., Petit, T., Peyer, K. E. \& Nelson, B. J. Targeted cargo delivery using a rotating nickel nanowire. Nanomedicine 8, 1074-1080 (2012).

32. Tierno, P. Recent advances in anisotropic magnetic colloids: realization, assembly and applications. Phys. Chem. Chem. Phys. 16, 23515-23528 (2014).

33. Cappallo, N., Lapointe, C., Reich, D. H. \& Leheny, R. L. Nonlinear microrheology of wormlike micelle solutions using ferromagnetic nanowire probes. Phys. Rev. E Stat. Nonlin. Soft Matter Phys. 76, 031505 (2007)
34. Rovner, J. B., Lapointe, C. P., Reich, D. H. \& Leheny, R. L. Anisotropic stokes drag and dynamic lift on cylindrical colloids in a nematic liquid crystal. Phys. Rev. Lett. 105, 228301 (2010)

35. Helgesen, G., Pieranski, P. \& Skjeltorp, A. T. Nonlinear phenomena in systems of magnetic holes. Phys. Rev. Lett. 64, 1425-1428 (1990).

36. Helgesen, G., Pieranski, P. \& Skjeltorp, A. T. Dynamic behavior of simple magnetic hole systems. Phys. Rev. A 42, 7271-7280 (1990).

37. Keshoju, K., Xing, H. \& Sun, L. Magnetic field driven nanowire rotation in suspension. Appl. Phys. Lett. 91, 123114 (2007).

38. McNaughton, B. H. et al. Compact sensor for measuring nonlinear rotationa dynamics of driven magnetic microspheres with biomedical applications. J. Magn. Magn. Mater. 321, 1648-1652 (2009).

39. Berret, J.-F., Sandre, O. \& Mauger, A. Size distribution of superparamagnetic particles determined by magnetic sedimentation. Langmuir 23, 2993-2999 (2007).

40. Fresnais, J. et al. Poly(acrylic acid)-coated iron oxide nanoparticles: Quantitative evaluation of the coating properties and applications for the removal of a pollutant dye. J. Colloid Interface Sci. 395, 24-30 (2013).

41. Chanteau, B., Fresnais, J. \& Berret, J.-F. Electrosteric enhanced stability of functional sub-10 $\mathrm{nm}$ cerium and iron oxide particles in cell culture medium. Langmuir 25, 9064-9070 (2009).

42. Berret, J.-F. et al. Stable oxide nanoparticle clusters obtained by complexation. J. Colloid Interface Sci. 303, 315-318 (2006).

43. Fresnais, J., Berret, J.-F., Frka-Petesic, B., Sandre, O. \& Perzynski, R. Electrostatic co-assembly of iron oxide nanoparticles and polymers: towards the generation of highly persistent superparamagnetic nanorods. Adv. Mater. 20, 3877-3881 (2008).

44. Colin, R., Yan, M., Chevry, L., Berret, J.-F. \& Abou, B. 3D rotational diffusion of micrometric wires using 2D video microscopy. Europhys. Lett. 97, 30008 (2012).

45. Tirado, M. M., Martinez, C. L. \& Delatorre, J. G. Comparison of theories for the translational and rotational diffusion-coefficients of rod-like macromolecules application to short DNA fragments. J. Chem. Phys. 81, 2047-2052 (1984).

46. Walker, L. M., Moldenaers, P. \& Berret, J.-F. Macroscopic response of wormlike micelles to elongational flow. Langmuir 12, 6309-6314 (1996).

47. Nussinovitch, A. Hydrocolloid Applications: Gum Technology in the Food and Other Industries (Springer Science + Business Media Dordrecht, 1997).

48. Safi, M. et al. Interactions between magnetic nanowires and living cells: uptake, toxicity, and degradation. ACS Nano 5, 5354-5364 (2011).

49. Leung, K. C. F. et al. Biological and magnetic contrast evaluation of shape-selective Mn-Fe nanowires. IEEE Trans. Nanobiosci. 8, 192-198 (2009)

50. Hillaireau, H. \& Couvreur, P. Nanocarriers' entry into the cell: relevance to drug delivery. Cell. Mol. Life Sci. 66, 2873-2896 (2009).

51. Doshi, N. \& Mitragotri, S. Needle-shaped polymeric particles induce transient disruption of cell membranes. J. R. Soc. Interface 7, S403-S410 (2010).

52. Shi, X., von dem Bussche, A., Hurt, R. H., Kane, A. B. \& Gao, H. Cell entry of one-dimensional nanomaterials occurs by tip recognition and rotation. Nat. Nanotechnol. 6, 714-719 (2011).

53. Cacchioli, A. et al. Cytocompatibility and cellular internalization mechanisms of $\mathrm{SiC} / \mathrm{SiO} 2$ nanowires. Nano Lett. 14, 4368-4375 (2014).

54. Champion, J. A. \& Mitragotri, S. Role of target geometry in phagocytosis. Proc. Natl Acad. Sci. USA 103, 4930-4934 (2006).

55. Liu, J. et al. Microrheology probes length scale dependent rheology. Phys. Rev. Lett. 96, 118104 (2006).

56. Castillo, M. et al. Local mechanical response of cells to the controlled rotation of magnetic nanorods. J. Biomed. Mater. Res. B Appl. Biomater. 102, 1779-1785 (2014).

\section{Acknowledgements}

K. Anselme, A. Baeza, A. Cebers, L. Chevry, E. Fodor, M.-A. Fardin, A. Hallou, F. Montel and P. Visco are acknowledged for fruitful discussions. Interns who participated in the research, C. Leverrier, A. Conte-Daban, C. Lixi, L. Carvhalo and R. Chan are also acknowledged. The author is grateful to the ImagoSeine facility (Jacques Monod Institute, Paris, France), and to France BioImaging infrastructure supported by the French National Research Agency (ANR-10-INSB-04, « Investments for the future »), and to L. Vitorazi, F. Mousseau and R. Le Borgne for the TEM images of the HeLa cells. The Laboratoire de Physico-chimie des Electrolytes et Nanosystémes Interfaciaux (UMR Université Pierre et Marie Curie-CNRS n ${ }^{\circ} 7612$ ) is acknowledged for providing us with the magnetic nanoparticles.

\section{Author contributions}

J.-F.B. conceived the project, developed, performed and supervised the experiments, carried out the data analysis and calculations, and wrote the paper.

\section{Additional information}

Supplementary Information accompanies this paper at http://www.nature.com/ naturecommunications

Competing financial interests: The author declares no competing financial interests. 
Reprints and permission information is available online at http://npg.nature.com/ reprintsandpermissions/

How to cite this article: Berret, J.-F. et al. Local viscoelasticity of living cells measured by rotational magnetic spectroscopy. Nat. Commun. 7:10134 doi: 10.1038/ncomms10134 (2016). (c) (i) This work is licensed under a Creative Commons Attribution 4.0 International License. The images or other third party material in this article are included in the article's Creative Commons license, unless indicated otherwise in the credit line; if the material is not included under the Creative Commons license, users will need to obtain permission from the license holder to reproduce the material. To view a copy of this license, visit http://creativecommons.org/licenses/by/4.0/ 